\title{
MHD stagnation point flow of micro nanofluid towards a shrinking sheet with convective and zero mass flux conditions
}

\author{
A. RAUF ${ }^{1}$, S.A. SHEHZAD ${ }^{1 *}$, T. HAYAT ${ }^{2,3}$, M.A. MERAJ $^{1}$, and A. ALSAEDI ${ }^{3}$ \\ ${ }^{1}$ Department of Mathematics, COMSATS Institute of Information Technology, Sahiwal 57000, Pakistan \\ ${ }^{2}$ Department of Mathematics, Quaid-I-Azam University, Islamabad 44000, Pakistan \\ ${ }^{3}$ Department of Mathematics, King Abdulaziz University, Jeddah 21589, Saudi Arabia
}

\begin{abstract}
In this article the stagnation point flow of electrically conducting micro nanofluid towards a shrinking sheet, considering a chemical reaction of first order is investigated. Involvement of magnetic field occurs in the momentum equation, whereas the energy and concentrations equations incorporated the influence of thermophoresis and Brownian motion. Convective boundary condition on temperature and zero mass flux condition on concentration are implemented. Partial differential equations are converted into the ordinary ones using suitable variables. The numerical technique is utilized to discuss the results for velocity, microrotation, temperature, and concentration fields.
\end{abstract}

Key words: micro nanofluid, shrinking sheet, MHD, chemical reaction.

\section{Introduction}

The analysis of fluid flow over stretching/shrinking surfaces has been of immense interest for the researchers in the fields of engineering and chemical process [1-4]. Especially the fluid flow generated by the surface is important in the extrusion and manufacturing process of material polymers, cooling systems, plastic industry, petrochemical industry, paper production, geophysical systems, power stations, chemical plants, air conditioning, refrigeration, etc. Out of all the above-mentioned systems, most attention was given to enhancing the energy generation and transfer of heat. Several methods have been proposed in this regard, but the techniques they employ are not suitable, due to lesser thermal conductivity of heat transfer fluid. Therefore, energy materials are introduced. These energy materials, known as nanomaterials, contain tiny particles of the same size as the de Broglie wave [5]. Therefore, nanoparticles have attracted researchers because of the abundance of applications thereof in technological and engineering processes. Nanoparticles in the base fluid, known as nanofluids, display thermophoresis and Brownian motion properties, which enhance the thermal performance and thermal conductivity of base fluids [6, 7]. Ultra-high heat transfer rates and extreme stability are the two main features of nanofluids [8] and do not cause problems such as erosion, sedimentation, and pressure drop.

Magnetohydrodynamics (MHD) is significant for chemistry, mathematics, physics, and engineering, and is applied in biological transportation, pumps, mixing of samples, cooling of strips, drug delivery, MHD generators, etc. External applied magnetic field is very helpful when controlling heat transfer and flow. As nanoparticles increase the thermal and electrical conductivity of nanofluids, making them liable to influence the magnetic

*e-mail: ali_qau70@yahoo.com field. Magnetic nanofluids are useful in nanocryosurgery, aerodynamics sensors, blood flow analysis, nuclear plants, artificial kidneys, and smartfluids for vibration damping. A number of researchers have discussed different models of hydromagnetic nanofluid flow over stretching surfaces [9-16].

Many biological fluids, as well as the fluids that are used in industrial applications such as printer inks, animal blood, detergents, paint, food stuff, polymer liquids, etc. change their flow characteristics when subjected to applied shear stress, and thus differ from Newtonian fluids. These materials are called non-Newtonian fluids. Researchers have discussed several non-Newtonian fluid flow models such as Maxwell fluid, power law fluid, second or third grade fluid, etc. Eringen $[17,18]$ introduced the theory of micropolar fluids for the first time. This theory deals with the intrinsic motion and local microstructure of the fluid particles and can be valuable when investigating the impact of polymer suspensions, colloidal solutions, biological and muddy fluids, etc. Furthermore, the impact of mass and heat transfer, combined with the impact of chemical reaction, has in the last few years been investigated with regard to possible applications in hydrometallurgical and chemical plants, including fruit-processing methods, freeze damage of crops, temperature distribution and growth of trees, and heat and mass transfer in cooling towers. Heat transfer due to surface convection and zero mass flux at a stretching/shrinking surface has gained significance in material dying, hot wiring, nuclear plants, transpiration process, production of glass fiber, heat exchangers, prevention of energy, etc.

The numerical solution of the problem of MHD stagnation point flow of micropolar fluid towards a moving sheet was presented by Ashraf and Bashir [19]. The extension of the above problem was performed by Rashidi et al. [20]. They added the term of mixed convection to the problem, and solved it analytically. Rauf et al. [21] numerically analyzed the MHD flow of micropolar fluid over a stretchable disk. The effects of a po- 
rous medium along with heat and mass transfer were studied. Ashraf and Wehgal [22] solved the problem of MHD flow of micropolar fluid confined between two fixed porous disks with heat transfer characteristics. Shehzad et al. [23] applied an analytical technique based on HAM to the problem of unsteady micropolar fluid and heat transfer influenced by a stretching sheet. Jalilpour et al. [24] applied HPM to the problem of MHD nanofluid flow over stretching sheet immersed in a porous medium. Pal et al. [25] analyzed stagnation point radiative flow of nanofluid over a surface with porous medium. The above-mentioned problem was extended to mixed convective nanofluid flow with chemical reaction by Pal and Mandal [26]. Hayat et al. [27] considered the problem of mixed convective flow over a stretching surface with chemical reaction. They employed the convective-type boundary condition at the surface of a sheet. Kuznetsov and Nield [28] considered the problem of boundary layer flow of nanofluid past a vertical plate. They implemented boundary conditions which imply that the flux of nanoparticles is zero at the boundary. The problem of Maxwell nanofluid flow over a stretching surface was solved by Hayat et al. [29]. Here, the Kuznetsov and Nield condition [28] of zero mass flux at the surface of a sheet was employed. The different flow problems under convective surface conditions have been modeled and addressed by Hayat et al. [30-32] and Imtiaz et al. [33].

Our main objective here is to find numerical solutions for MHD stagnation point flow of an incompressible, electrically conducting flow of micro nanofluid over a heated shrinking sheet. The sheet obeys the convective condition on temperature and the zero-mass condition. The variations of individual parameters of interest are examined.

\section{Problem formulation}

Here, two-dimensional laminar incompressible stagnation point flow of an electrically conducting micro nanofluid impinging in normal direction over a heated shrinking sheet is considered. We investigate the impact of magnetic field of strength $B_{0}$. Magnetic field is utilized in transverse direction of the flow field. The governing equations are:

$$
\begin{gathered}
\frac{\partial u}{\partial x}+\frac{\partial v}{\partial y}=0 \\
\rho\left(u \frac{\partial u}{\partial x}+v \frac{\partial u}{\partial y}\right)=U \frac{d U}{d x}+(\mu+k) \frac{\partial^{2} u}{\partial y^{2}}+ \\
+k \frac{\partial v}{\partial y}+\sigma_{e} B_{0}{ }^{2}(U-u) \\
\rho j\left(u \frac{\partial v}{\partial x}+v \frac{\partial v}{\partial y}\right)=\gamma \frac{\partial^{2} v}{\partial y^{2}}-k\left(2 v+\frac{\partial u}{\partial y}\right) \\
u \frac{\partial T}{\partial x}+v \frac{\partial T}{\partial y}=\alpha \frac{\partial^{2} T}{\partial y^{2}}+\tau\left(D_{B} \frac{\partial C}{\partial y} \frac{\partial T}{\partial y}+\frac{D_{T}}{D_{\infty}}\left(\frac{\partial T}{\partial y}\right)^{2}\right),
\end{gathered}
$$

$$
u \frac{\partial C}{\partial x}+v \frac{\partial C}{\partial y}=D_{B} \frac{\partial^{2} C}{\partial y^{2}}+\frac{D_{T}}{T_{\infty}} \frac{\partial^{2} T}{\partial y^{2}}-k_{1}\left(C-C_{\infty}\right),
$$

where: $u$ and $v$ are the velocity components in the $\mathrm{x}$ and $\mathrm{y}$ directions respectively, $p$ is the pressure, $v$ is the microrotation, $\rho$ is the density, $\mu$ is the viscosity, $k$ is the vortex viscosity, $j$ is the microinertia density, $\gamma$ is the spin gradient viscosity, $\sigma_{e}$ is the electrical conductivity of fluid, $\alpha$ is the thermal diffusivity of fluid, $\tau=\frac{(\rho c)_{p}}{(\rho c)_{f}}$ is the ratio of nanoparticle heat capacity and the base fluid heat capacity, $D_{B}$ is the Brownian diffusion coefficient, $D_{T}$ is the thermophoretic diffusion coefficient, and $k_{1}$ is the reaction coefficient. The boundary conditions are:

$$
\left\{\begin{array}{l}
u(x, 0)=b x, v(x, 0)=0, v(x, 0)=0, \\
-k \frac{\partial T}{\partial y}=h_{f}\left(T_{f}-T\right), \\
D_{B} \frac{\partial C}{\partial y}+\frac{D_{B}}{T_{\infty}} \frac{\partial T}{\partial y}=0 \\
u(x, \infty)=U=a x, v(x, \infty)=0, \\
T(x, \infty)=T_{\infty}, C(x, \infty)=C_{\infty},
\end{array}\right.
$$

where $b<0$ corresponds to the sheet shrinking rate and $h_{f}$ is the heat transfer coefficient. Considering the similarity transformations:

$$
\left\{\begin{array}{l}
u=a x f^{\prime}(\eta), v=-\sqrt{a v} f(\eta), \\
v_{2}=-a \sqrt{\frac{a}{v}} x g(\eta), \eta=y \sqrt{\frac{a}{v}}, \\
\theta(\eta)=\frac{T-T_{\infty}}{T_{f}-T_{\infty}}, \phi(\eta)=\frac{C-C_{\infty}}{C_{\infty}},
\end{array}\right.
$$

equations (1-5) become

$$
\begin{gathered}
\left(1+R_{1}\right) f^{\prime \prime \prime}-R_{1} g^{\prime}+M^{2}\left(1-f^{\prime}\right)+1-f^{\prime 2}+f f^{\prime \prime}=0 \\
C_{1} g^{\prime \prime}+R_{1} A_{1}\left(f^{\prime \prime}-2 g\right)-f^{\prime} g+f g^{\prime}=0 \\
\theta^{\prime \prime}+\operatorname{Pr} f \theta^{\prime}+\operatorname{Pr} N_{b} \theta^{\prime} \phi^{\prime}+\operatorname{Pr} N_{t} \theta^{\prime 2}=0 \\
\phi^{\prime \prime}+\operatorname{Sc} f \phi^{\prime}+\left(\frac{N_{t}}{N_{B}}\right) \theta^{\prime \prime}-S c \gamma_{1} \phi=0
\end{gathered}
$$

Here, $M=\sqrt{\frac{\sigma_{e} B_{0}^{2}}{\rho \alpha}}$ denotes the magnetic parameter, $R_{1}=\frac{k}{\mu}$ the vortex viscosity parameter, $C_{1}=\frac{\gamma}{\mu j}$ the spin gradient viscosity, $A_{1}=\frac{\mu}{\rho j a}$ microinertia density parameter, $\operatorname{Pr}=\frac{\mu c_{p}}{k_{0}}$ the Prandtl number, $N_{b}=\frac{(\rho c)_{p} D_{B} C_{\infty}}{(\rho c)_{f} v}$ the Brownian motion parameter, $N_{t}=\frac{(\rho c)_{p} D_{T}\left(T_{f}-T_{\infty}\right)}{(\rho c)_{f} v T_{\infty}}$ the thermophoresis parameter, $S c=\frac{v}{D_{B}}$ the Schmidt number, and $\gamma_{1}=\frac{k_{1}}{a}$ the chemical reaction parameter. 
The dimensionless boundary conditions are:

$$
\left\{\begin{array}{l}
f^{\prime}(0)=N, f(0)=0, g(0)=0, \\
\theta^{\prime}(0)=-B i(1-\theta(0)), \\
N t \theta^{\prime}(0)+N b \phi^{\prime}(0)=0, \\
f^{\prime}(\infty)=1, g(\infty)=0, \\
\theta(\infty)=0, \phi(\infty)=0,
\end{array}\right.
$$

where $B_{i}=\frac{h_{f}}{k} \sqrt{\frac{v}{a}}$ is the heat transfer Biot number and $N=\frac{b}{a}$ is the shrinking parameter.

\section{Numerical solution}

The Runge-Kutta-Fehlberg (RKF45) method is very helpful when solving $\frac{d y}{d x}=f(x, y), y\left(x_{l}\right)=y_{l}$. The procedure involves suitable step size which guarantees the accurateness in solution of the initial value problem. Every proper step contains two different types of approximations to the solution, which are computed and compared. When answers match closely, the approximation is valid. If answers are not accurate enough, then the step size is decreased. If the answers meet more than the significant digits, the step size is incremented. In each step the following six steps are required:

$$
\begin{aligned}
& I_{1}=h f\left(x_{m} y_{m}\right), \\
& I_{2}=h f\left(x_{m}+\frac{1}{4} h, y_{m}+\frac{1}{4} I_{m}\right), \\
& I_{3}=h f\left(\begin{array}{l}
x_{m}+\frac{3}{8} h, y_{m} \\
+\frac{3}{32} I_{m}+\frac{9}{32} I_{2}
\end{array}\right), \\
& I_{4}=h f\left(\begin{array}{l}
x_{m}+\frac{12}{13} h, y_{m}+\frac{1932}{2197} I_{m} \\
-\frac{7200}{2197} I_{2}+\frac{7296}{2197} I_{3}
\end{array}\right), \\
& I_{5}=h f\left(\begin{array}{c}
x_{m}+h, y_{m}+\frac{439}{216} I_{1} \\
-8 I_{2}+\frac{3680}{513} I_{3}-\frac{845}{4104} I_{4}
\end{array}\right), \\
& I_{6}=h f\left(\begin{array}{l}
1 \\
x_{m}+\frac{1}{2} h, y_{m} \\
-\frac{8}{27} I_{1}+2 I_{2}-\frac{3544}{2565} I_{3} \\
+\frac{1859}{4104} I_{4}-\frac{11}{40} I_{5}
\end{array}\right) .
\end{aligned}
$$

The approximation of order 4 to the solution is:

$$
y_{m+1}=x_{m}+\frac{25}{216} I_{1}+\frac{1408}{2565} I_{3}+\frac{2197}{4101} I_{4}-\frac{1}{5} I_{5} .
$$

A better approximation of order 5 to the solution is given by:

$$
\begin{aligned}
& z_{m+1}=y_{m}+\frac{16}{135} I_{m}+\frac{6656}{12825} I_{3}+ \\
& +\frac{28561}{56430} I_{4}-\frac{9}{50} I_{5}+\frac{2}{55} I_{6} .
\end{aligned}
$$

Finally, $d h$ stands for optimal step size and is obtained by multiplying $h$ with a scalar $d$ Here, $d$ is determined by:

$$
d=0.84\left(\frac{\text { tol } h}{2\left|z_{m+1}-y_{m+1}\right|}\right)^{\frac{1}{4}}
$$

where $t o l$ stands for the error tolerance.

A finite difference technique based on RKF45 method with a shooting technique [34-36] is implemented to obtain the numerical solution of the systems (8-11) with the corresponding boundary conditions (12). The following are set:

$$
\begin{aligned}
& z_{1}=f, z_{2}=f^{\prime}, z_{3}=f^{\prime \prime}, \\
& z_{4}=g, z_{5}=g^{\prime}, z_{6}=\theta, \\
& z_{7}=\theta^{\prime}, z_{8}=\phi, z_{9}=\phi^{\prime},
\end{aligned}
$$

where prime stands for the derivative with respect to $\eta$. Using (17) into (8-11), we obtain the reduced first-order system of differential equations:

$$
\begin{gathered}
\left(1+R_{1}\right) z_{3}{ }^{\prime}-R_{1} z_{5}+M^{2}\left(1-z_{2}\right)+1-z_{2}^{2}+z_{1} z_{3}=0 \\
C_{1} z_{5}^{\prime}+R_{1} A_{1}\left(z_{3}-2 z_{4}\right)-z_{2} z_{4}+z_{1} z_{5}=0 \\
z_{7}^{\prime}+\operatorname{Pr} z_{1} z_{7}+\operatorname{Pr} N_{b} z_{7} z_{9}+\operatorname{Pr} N_{t} z^{2}=0 \\
z_{9}^{\prime}+\operatorname{Sc} z_{1} z_{9}+\left(\frac{N_{t}}{N_{B}}\right) z_{7}^{\prime}-S c \gamma_{1} z_{8}=0
\end{gathered}
$$

The boundary conditions (12) become

$$
\left\{\begin{array}{l}
z_{1}(0)=0, z_{2}(0)=N, z_{3}(0)=a_{1}, \\
z_{4}(0)=0, z_{5}(0)=a_{2}, \\
z_{7}(0)=-B_{i}\left(1-z_{6}(0)\right), z_{8}(0)=a_{3}, \\
N t z_{7}(0)+N b z_{9}(0)=0
\end{array}\right.
$$

The Newton-Raphson algorithm and the shooting method are used to guess the conditions $a_{1}, a_{2}$, and $a_{3}$ in (22). Finally, the problem is integrated to obtain the boundary conditions at $\eta=0$. The convergence criteria are set to at least $10^{-7}$.

\section{Results and discussion}

This section is devoted to illustrating our findings in graphical, as well as tabular forms. The dimensionless parameters 


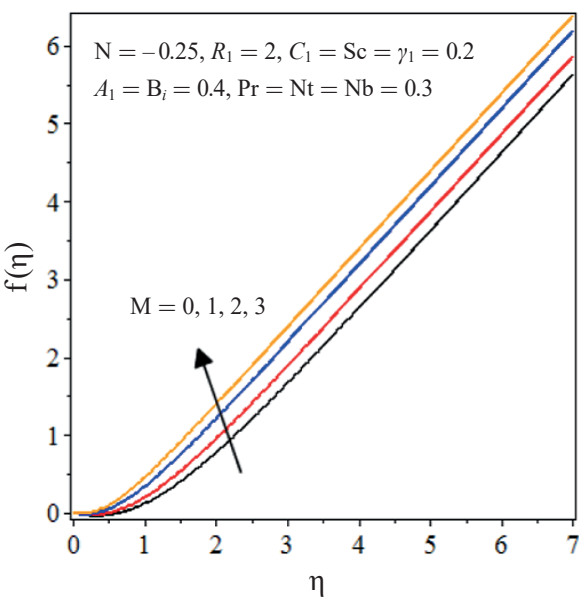

Fig. 1. $f(\eta)$ for different values of $M$

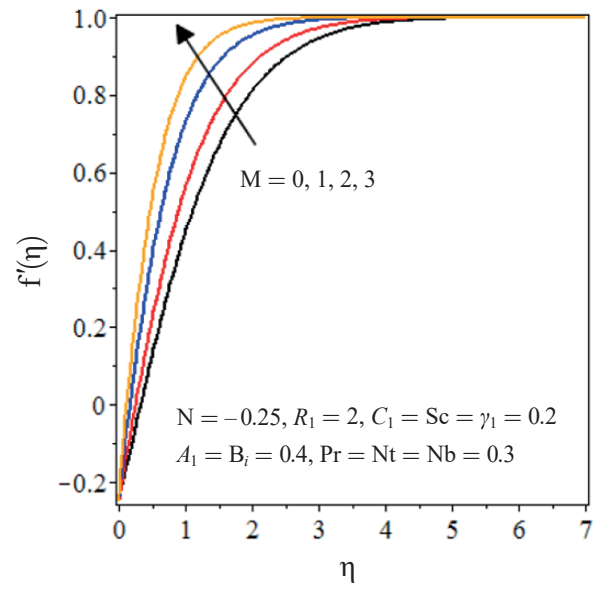

Fig. 2. $f^{\prime}(\eta)$ for different values of $M$

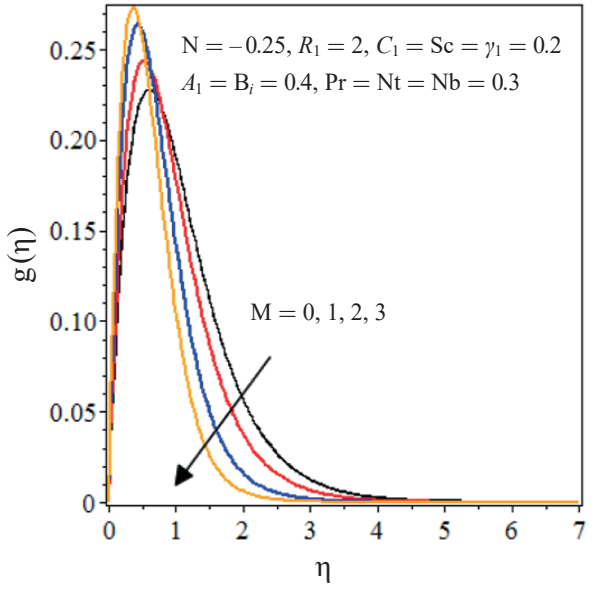

Fig. 3. $g(\eta)$ for different values of $M$ of our interests are the micropolar parameters $R_{1}, C_{1}$, and $A_{1}$, magnetic parameter $M$, shrinking parameter $N$, Prandtl number Pr, heat transfer Biot number $B_{i}$, Schmidt number $S c$, thermophoretic parameter $\mathrm{Nt}$, Brownian motion parameter $\mathrm{Nb}$, and chemical reaction parameter $\gamma_{1}$. To have the best knowledge of the physics of our model, we chose to describe shear and couple stresses and heat and mass transfer rates at the sheet, considering different values of the physical parameters. We fix $R_{1}=2, C_{1}=0.2, A_{1}=0.4, M=0.5, N=-0.25, \operatorname{Pr}=0.3$, $S c=0.2, N t=0.3, N b=0.3, B_{i}=0.4$, and $\gamma_{1}=0.2$ into our computation procedure, altering one parameter at a time, as discussed through graphs and tables. We adjusted $\eta_{\infty}=7,10,15$ in order to have asymptotic behavior of velocity, microrotation, temperature, and concentration profiles.

Figures 1-3 are drawn to explore the behavior of magnetic parameter $M$ on velocity and microrotation. Here, $M=0$ shows the hydrodynamic flow and $M(>0)$ represents hydromagnetic flow. Increasing $M$ results in an enhancement in normal velocity profiles $f(\eta)$ and streamwise velocity profiles $f^{\prime}(\eta)$. A reverse flow region can be seen near the surface because of the shrinking of the sheet [19]. Fig. 1 shows that large $M$ can be helpful to stop the reverse flow phenomenon. The imposed magnetic field produces a frictional force called the Lorentz force, which offers a resistance in a flow field, and due to its velocity, the boundary layer pushes towards the wall of the sheet, as shown in Fig. 2. An increase in the magnetic parameter causes a reduction in microrotation profiles, as described in Fig. 3.

Figures 4-6 are presented to investigate the impact of the shrinking parameter on $f(\eta), f^{\prime}(\eta)$, and $g(\eta)$. It is noted from Figs. 4 and 5 that $f(\eta)$ and $f^{\prime}(\eta)$ decrease by enhancing $N$. The reverse flow in the vicinity of surface of the sheet is also observed for increased values in magnitude of $N$. Fig. 6 illustrates the influence of $N$ on microrotation profiles $g(\eta)$. The profiles are increased due to the enhanced values of magnitude of $N$. Fig. 7 explores the behavior of $R_{1}, C_{1}$, and $A_{1}$ in microrotation. Influence of different values of micropolar parameters causes an enhancement in profiles $g(\eta)$. Fig. 8 illustrates the effect of Prandtl number on temperature profiles. Physically, the Prandtl number is inversely proportional to the thermal diffusivity. Hence, larger values of Pr produce weaker thermal diffusivity. This corresponds to a reduction in both temperature and the

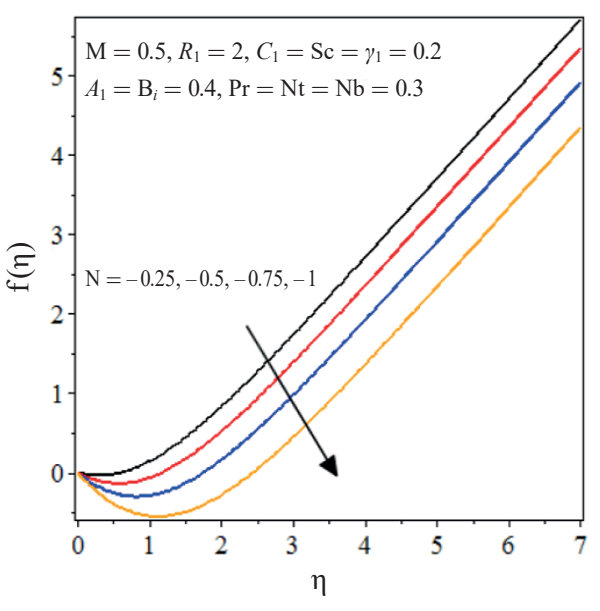

Fig. 4. $f(\eta)$ for different values of $N$

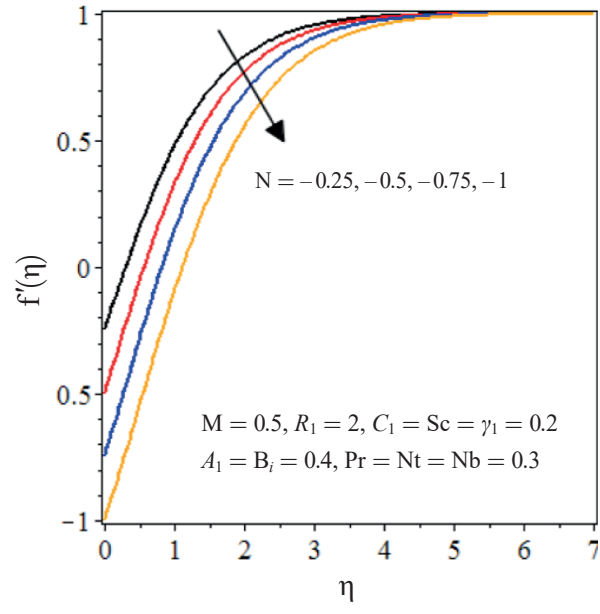

Fig. 5. $f^{\prime}(\eta)$ for different values of $N$

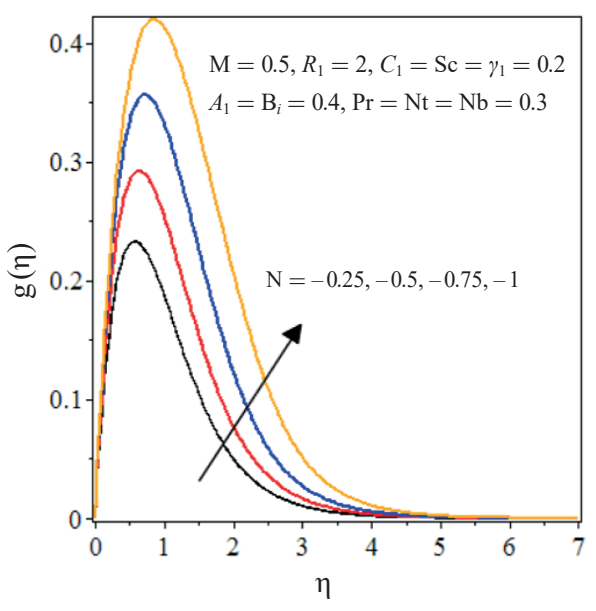

Fig. 6. $g(\eta)$ for different values of $N$ 


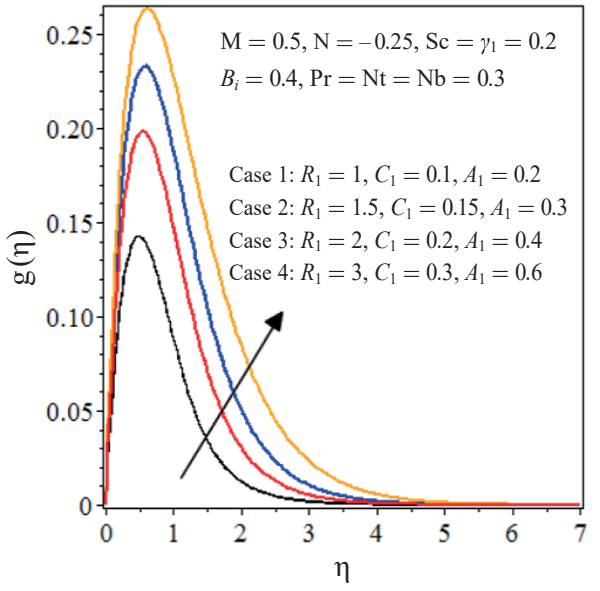

Fig. 7. $g(\eta)$ for different four cases

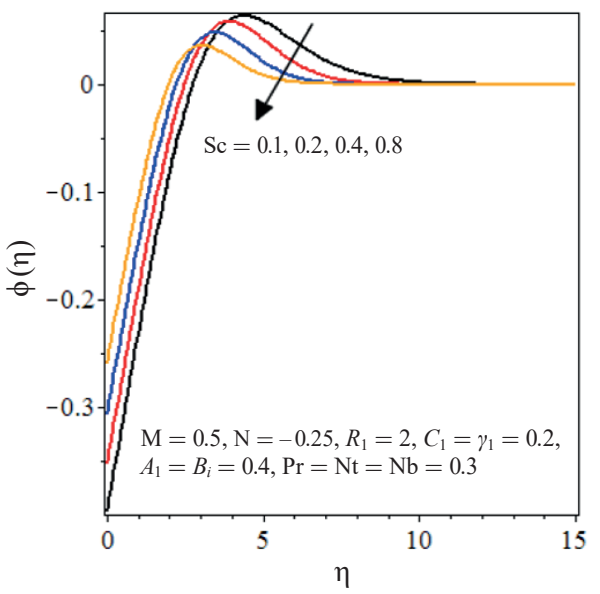

Fig. 10. $\phi(\eta)$ for different values of $S c$

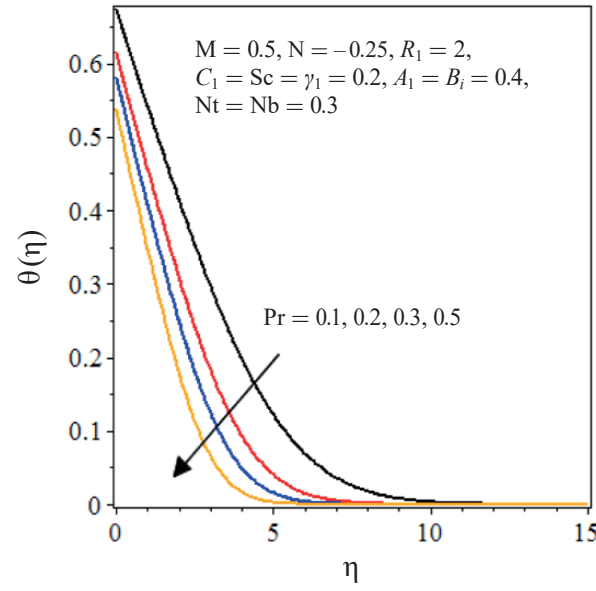

Fig. 8. $\theta(\eta)$ for different values of $\operatorname{Pr}$

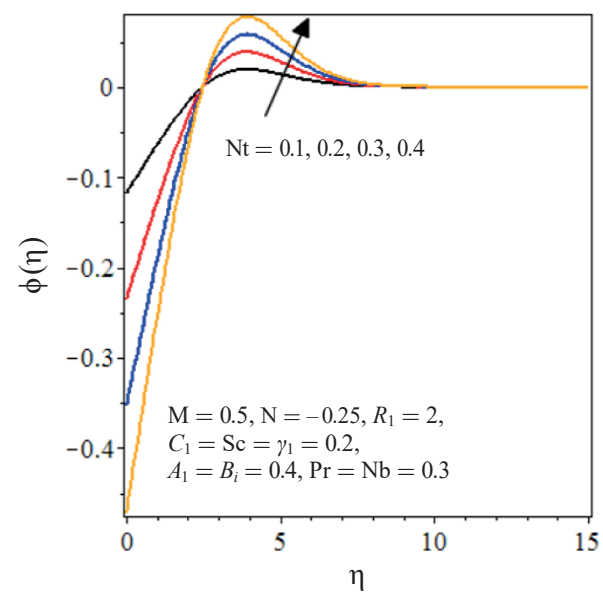

Fig. 11. $\phi(\eta)$ for different values of $N t$

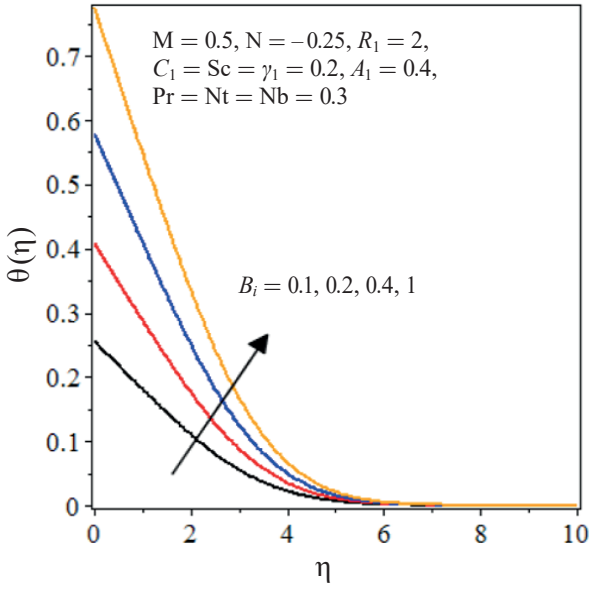

Fig. 9. $\theta(\eta)$ for different values of $B_{i}$

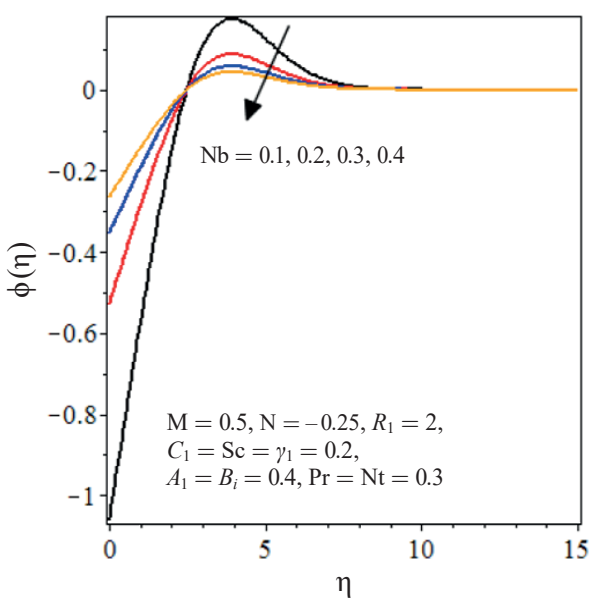

Fig. 12. $\phi(\eta)$ for different values of $N b$ associated boundary layer thickness. Fig. 9 is plotted to explore the impact of $B_{i}$ on $\theta(\eta)$. Physically, the Biot number is the ratio of internal thermal resistance at the surface of the body to the boundary layer thermal resistance. Therefore, enhancing the values of $B_{i}$ shows an increase in temperature profiles and its related boundary layer thickness. Fig. 10 is sketched for a better understanding of the impact of $S c$ on concentration profiles $\phi(\eta)$. Physically, Schmidt number is inversely proportional to the mass diffusion, therefore an increase in Sc causes a reduction in nanoparticle concentration profiles, as well as in related boundary layer thickness.

Figures 11-12 are designed to depict the effect of thermophoretic and Brownian motion parameters on $\phi(\eta)$. In thermophoresis, the small particles are pushed away from the hot surface and are driven towards a cold surface, therefore increasing the values of $N t$, which causes an increase in nanoparticle concentration profiles (Fig. 11). The Brownian motion comes into play due to the zig-zag movement of nanoparticles. Such motion then results in an increase of kinetic energy of particles, and hence the collision between the particles increases. Therefore, the Brownian motion is affected by the increasing values of $\mathrm{Nb}$, which then reduces $\phi(\eta)$ with the relevant boundary layer thickness, as shown in Fig. 12. Figure 13 shows that the profiles $\phi(\eta)$ and the appropriate boundary layer thickness decrease with de-

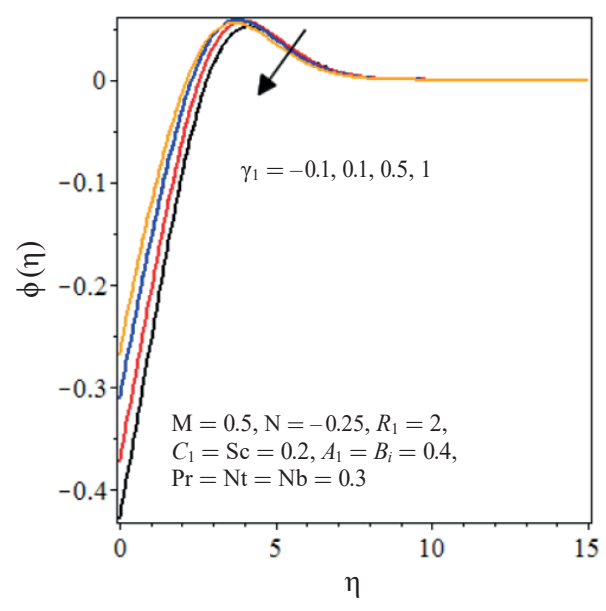

Fig. 13. $\phi(\eta)$ for different values of $\gamma_{1}$ 
structive chemical reaction $\left(\gamma_{1}>0\right)$, whereas an opposite trend is seen for generative chemical reaction $\left(\gamma_{1}<0\right)$.

Table 1 is formed to describe the arbitrary values of micropolar parameters [19]. Table 2 is drawn to present the impact of the magnetic parameter, shrinking parameter and micropolar parameters on shear and couple stresses. It is noted that shear stresses intensify with an increase in $M$, while a reverse trend is observed for $R_{1}, C_{1}$, and $A_{1}$. An increase in the shear stresses is seen for $0.25<-N<0.75$, whereas the opposite behavior can be noted for $0.75<-N<1$. The couple stresses are increasing for increasing values of micropolar parameters, magnetic parameter, and shrinking parameter, as shown in Table 2. Table 3 displays the effects of heat transfer rate for various values of Pr and $B_{i}$. Rising values of the Prandtl number and heat transfer Biot number lead to an increase in heat transfer rate at the sheet. Table 4 presents the impact of the Schmidt number, thermophoretic parameter, Brownian motion parameter and destructive/ generative chemical reaction parameter on $\phi^{\prime}(\eta)$. It is seen that the mass transfer rate is a decreasing function of $S c, N b$, and $\gamma_{1}$. However, the opposite trend is noted for larger values of $N t$. Table 5 was made to present the validity of the numerical results. It is apparent that the results are compared well with the previously published literature work.

Table 1

Different values of $R_{1}, C_{1}$, and $A_{1}$ for the four cases discussed

\begin{tabular}{|c|c|c|c|}
\hline Case No. & $R_{1}$ & $C_{1}$ & $A_{1}$ \\
\hline 1 & 1 & 0.1 & 0.2 \\
\hline 2 & 1.5 & 0.15 & 0.3 \\
\hline 3 & 2 & 0.2 & 0.4 \\
\hline 4 & 3 & 0.3 & 0.6 \\
\hline
\end{tabular}

Table 2

Shear and couple stresses at sheet for different values of $M, N$, and four Cases of $R_{1}, C_{1}$, and $A_{1}$

\begin{tabular}{|c|c|c|c|c|}
\hline$M$ & $-N$ & Case No. & $\left(1+R_{1}\right) f^{\prime \prime}(0)$ & $g^{\prime}(0)$ \\
\hline 0 & 0.25 & 3 & 0.72994 & 0.96967 \\
\hline 1 & & & 0.99312 & 1.18024 \\
\hline 2 & & & 1.54858 & 1.54426 \\
\hline 3 & & & 2.19657 & 1.87129 \\
\hline 0.5 & 0.25 & 3 & 0.80299 & 1.03122 \\
\hline & 0.5 & & 0.85992 & 1.18253 \\
\hline & 0.75 & & 0.86544 & 1.28875 \\
\hline & 1 & & 0.80069 & 1.31458 \\
\hline 0.5 & 0.25 & 1 & 1.04855 & 0.68654 \\
\hline & & 2 & 0.90768 & 0.89683 \\
\hline & & 3 & 0.80299 & 1.03122 \\
\hline & & 4 & 0.66129 & 1.18097 \\
\hline
\end{tabular}

Table 3

Heat transfer rate at the sheet for different values of $\operatorname{Pr}$ and $B_{i}$

\begin{tabular}{|c|c|c|}
\hline $\operatorname{Pr}$ & $B_{i}$ & $-\theta^{\prime}(0)$ \\
\hline 0.1 & 0.4 & 0.13516 \\
\hline 0.2 & & 0.15499 \\
\hline 0.3 & & 0.16835 \\
\hline 0.5 & & 0.18529 \\
\hline 0.3 & 0.1 & 0.07444 \\
\hline & 0.2 & 0.11853 \\
\hline & 0.4 & 0.16835 \\
\hline & 1 & 0.22502 \\
\hline
\end{tabular}

Table 4

Mass transfer rate at the sheet for different values of $S c, N t, N b$, and $\gamma_{1}$

\begin{tabular}{|c|c|c|c|c|}
\hline$S c$ & $N t$ & $N b$ & $\gamma_{1}$ & $\phi^{\prime}(0)$ \\
\hline 0.1 & 0.3 & 0.3 & 0.2 & 0.16848 \\
\hline 0.2 & & & & 0.16835 \\
\hline 0.4 & & & & 0.16817 \\
\hline 0.8 & & & & 0.16794 \\
\hline 0.2 & 0.1 & 0.3 & 0.2 & 0.05619 \\
\hline & 0.2 & & & 0.11231 \\
\hline & 0.3 & & & 0.16835 \\
\hline & 0.4 & & & 0.22432 \\
\hline 0.2 & 0.3 & 0.1 & 0.2 & 0.50505 \\
\hline & & 0.2 & & 0.25252 \\
\hline & & 0.3 & & 0.16835 \\
\hline & & 0.4 & & 0.12626 \\
\hline 0.2 & 0.3 & 0.3 & -0.1 & 0.16862 \\
\hline & & & 0.1 & 0.16842 \\
\hline & & & 0.5 & 0.16818 \\
\hline & & & 1 & 0.16798 \\
\hline
\end{tabular}

Table 5

Comparison of numerical values of shear and couple stresses at sheet for various values of $M[19]$

\begin{tabular}{|c|c|c|c|c|}
\hline \multirow{2}{*}{$M$} & \multicolumn{2}{|c|}{$\left(1+R_{1}\right) f^{\prime \prime}(0)$} & \multicolumn{2}{c|}{$g^{\prime}(0)$} \\
\cline { 2 - 5 } & $\begin{array}{c}\text { results from } \\
{[19]}\end{array}$ & $\begin{array}{c}\text { present } \\
\text { study }\end{array}$ & $\begin{array}{c}\text { results from } \\
{[19]}\end{array}$ & $\begin{array}{c}\text { present } \\
\text { study }\end{array}$ \\
\hline 0 & 0.52887 & 0.52869 & 1.14119 & 1.14243 \\
\hline 1 & 0.85965 & 0.85932 & 1.62744 & 1.62940 \\
\hline 2 & 1.49003 & 1.48955 & 2.37378 & 2.37714 \\
\hline 3 & 2.20069 & 2.20027 & 3.03622 & 3.04127 \\
\hline
\end{tabular}




\section{Conclusion}

The following conclusions can be drawn from the presented analysis:

1) Couple stresses are enhanced by increasing $M, N, R_{1}$, $C_{1}$, and $D_{1}$.

2) $\operatorname{Pr}$ and $B_{i}$ increase $\theta^{\prime}(\eta)$.

3) Mass transfer rate enhances with the increase of $N t$, whereas a reverse trend is noted in case of increasing $S c, N b$, and $\gamma_{1}$.

4) Temperature profiles and thermal boundary layer thicknesses are decreasing functions of $\mathrm{Pr}$, while the opposite behavior is seen in case of enhancing the values of $B_{i}$.

5) Concentration profile decreases for increasing values of the Schmidt number and Brownian motion parameter. On the other hand, the profiles increase for larger values of the thermophoretic parameter.

\section{REFERENCES}

[1] S.A. Shehzad, Z. Abdullah, A. Alsaedi, F.M. Abbasi, and T. Hayat, "Thermally radiative three-dimensional flow of Jeffrey nanofluid with internal heat generation and magnetic field", J. Magnet. Magnet. Mater. 397, 108-114 (2016)

[2] L. Zheng, J. Niu, X. Zheng, and L. Ma, "Dual solutions for flow and Radiative heat transfer of micropolar fluid over stretching/ shrinking sheet”, Int. J. Heat Mass Transf. 55 (25-26), 7577-7586 (2012).

[3] B.J. Gireesha, A.J. Chamkha, S. Manjunatha, and C.S. Bagewadi, "Mixed convective flow of a dusty fluid over a vertical stretching sheet with non-uniform heat source/sink and radiation", Int. J. Numer. Meth. Heat Fluid Flow 23 (4), 598-612 (2013).

[4] B.J. Gireesha, G.K. Ramesh, M.S. Abel, and C.S. Bagewadi, "Boundary layer flow and heat transfer of a dusty fluid flow over a stretching sheet with non-uniform heat source/sink", Int. J. Multiphase Flow 37 (8), 977-982 (2011).

[5] A. Sharma, V.V. Tyagi, C.R. Chen, and D. Buddhi, "Review on thermal energy storage with phase change materials and application", Renew. Sustain. Energy Rev. 13 (2), 318-345 (2009).

[6] S.U.S. Choi and J.A. Eastman, "Enhancing thermal conductivity of fluids with nanoparticles", ASME International Engineering Congress and Exposition, San Francisco 66, 99-105 (1995).

[7] D.K. Devendiran and V.A. Amirtham, "A review on preparation, characterization, properties and applications on nanofluids", Renew. Sustain. Energy Rev. 60, 21-40 (2016).

[8] M.M. Rahman, W.A. Al-Mazroui, F.S. Al-Hatmi, M.A. Al-Lawatia, and I.A. Eltayeb, "The role of a convective surface in models of the radiative heat transfer in nanofluids", Nuclear Eng. Design 275, 382-392 (2014).

[9] S.A. Shehzad, T. Hayat, and A. Alsaedi, "Influence of convective heat and mass conditions in MHD flow of nanofluid", Bull. Pol. Ac.: Tech. 63 (2), 465-474 (2015).

[10] S.A. Shehzad, Z. Abdullah, F.M. Abbasi, T. Hayat, and A. Alsaedi, "Magnetic field effect in three-dimensional flow of an Oldroyd-B nanofluid over a radiative surface", J. Magnet. Magnet. Mater. 399, 97-108 (2016).
[11] T. Hayat, T. Muhammad, B. Ahmad, and S.A. Shehzad, "Impact of magnetic field in three-dimensional flow of Sisko nanofluid with convective condition", J. Magnet. Magnet. Mater. 413, 1-8 (2016).

[12] M.M. Rashidi, N.V. Ganesh, A.K.A. Hakeem, and B. Ganga, "Buoyancy effect on MHD flow of nanofluid over a stretching sheet in the presence of thermal radiation", J. Mol. Liq. 198, 234-238 (2014).

[13] T. Hayat, T. Muhammad, S.A. Shehzad, G.Q. Chen, and I.A. Abbas, "Interaction of magnetic field in flow of Maxwell nanofluid with convective effect", J. Magnet. Magnet. Mater. 389, 48-55 (2015).

[14] T. Hayat, M. Imtiaz, and A. Alsaedi, "Unsteady flow of nanofluid with double stratification and magnetohydrodynamics", Int. J. Heat Mass Transf. 92, 100-109 (2016).

[15] T. Hayat, M. Waqas, M.I. Khan, and A. Alsaedi, "Analysis of thixotropic nanomaterial in a doubly stratified medium considering magnetic field effects", Int. J. Heat Mass Transf. 102, 1123-1129 (2016)

[16] T. Hayat, M.I. Khan, M. Waqas, T. Yasmeen, and A. Alsaedi, "Viscous dissipation effect in flow of magnetonanofluid with variable properties", J. Mol. Liq. 222, 47-54 (2016).

[17] A.C. Eringen, "Simple micropolar fluids", Int. J. Eng. Sci. 2, 205-217 (1964).

[18] A.C. Eringen, "Theory of micropolar fluids", J. Appl. Math. Mech. 16, 1-18 (1966).

[19] M. Ashraf, and S. Bashir, "Numerical simulation of MHD stagnation point flow and heat transfer of a micropolar fluid towards a heated shrinking sheet”, Int. J. Numer. Methods Fluids 69 (2), 384-398 (2012).

[20] M.M. Rashidi, M. Ashraf, B. Rostami, M.T. Rastegari, and S. Bashir, "Mixed convection boundary-layer flow of a micropolar fluid towards a heated shrinking sheet by homotopy analysis method", Thermal Sci. 20 (1), 21-34 (2016).

[21] A. Rauf, M. Ashraf, K. Batool, T. Hussain, and M.A. Meraj, "MHD flow of a micropolar fluid over a stretchable disk in a porous medium with heat and mass transfer", AIP Adv. 5, 077156 (2015).

[22] M. Ashraf and A.R. Wehgal, "MHD flow and heat transfer of a micropolar fluid between two porous disks", Appl. Math. Mech. 33 (1), 51-64 (2012).

[23] S.A. Shehzad, M. Waqas, A. Alsaedi, and T. Hayat, "Flow and heat transfer over an unsteady stretching sheet in a micropolar fluid with convective boundary conditions", J. Appl. Fluid Mech. 9 (3), 1437-1445 (2016).

[24] B. Jalilpour, S. Jafarmadar, D.D. Ganji, A.B. Shotorban, and H. Taghavifar, "Heat generation/absorption on MHD stagnation flow of nanofluid towards a porous stretching sheet with prescribed surface heat flux", J. Mol. Liq. 195, 194-204 (2014).

[25] D. Pal, G. Mandal, and K. Vajravelu, "Flow and heat transfer of nanofluids at a stagnation point flow over a stretching/shrinking surface in a porous medium with thermal radiation", Appl. Math. Comput. 238, 208-224 (2014).

[26] D. Pal, and G. Mandal, "Influence of thermal radiation of mixed convection heat and mass transfer stagnation-point flow in nanofluids over stretching/shrinking sheet in a porous medium with chemical reaction", Nuclear Eng. Design 273, 644-652 (2014).

[27] T. Hayat, M.B. Ashraf, S.A. Shehzad, and A. Alsaedi, "Mixed convection flow of Casson nanofluid over a stretching sheet with convectively heated chemical reaction and heat source/sink", J. Appl. Fluid Mech. 8 (4), 803-813 (2015). 
[28] A.V. Kuznetsov, and D.A. Nield, "Natural convective boundary-layer flow of a nanofluid pas a vertical plate: A revised-model", Int. J. Thermal Sci. 77, 126-129 (2014).

[29] T. Hayat, T. Muhammad, S.A. Shehzad, and A. Alsaedi, "Three-dimensional boundary layer flow of Maxwell nanofluid: mathematical model", Appl. Math. Mech. 36 (6), 747-762 (2015).

[30] T. Hayat, S. Farooq, A. Alsaedi, and B. Ahmad, "Hall and radial magnetic field effects on radiative peristaltic flow of Carreau-Yasuda fluid in a channel with convective heat and mass transfer", J. Magnet. Magnet. Mater. 412, 207-216 (2016).

[31] T. Hayat, M. Imtiaz, and A. Alsaedi, "MHD 3D flow of nanofluid in presence of convective conditions", J. Mol. Liq. 212, 203-208 (2015).

[32] T. Hayat, Y. Saeed, S. Asad, and A. Alsaedi, "Convective heat and mass transfer in flow by an inclined stretching cylinder", J. Mol. Liq. 220, 573-580 (2016).
[33] M. Imtiaz, T. Hayat, A. Alsaedi, and B. Ahmad, "Convective flow of carbon nanotubes between rotating stretchable disks with thermal radiation effects", Int. J. Heat Mass Transf. 101, 948-957 (2016).

[34] W.M.K.A.D. Zaimi, B. Bidin, N.A.A. Bakar, and R.A. Hamid, "Applications of Runge-Kutta-Fehlberg method and shooting technique for solving classical Blasius equation", World Appl. Sci. J. 17, 10-15 (2012).

[35] A.K. Jhankal and M. Kumar, "Magnetohydrodynamic (MHD) plane poiseuille flow with variable viscosity and unequal wall temperature", Iran. J. Chem. Eng. 11 (1), 63-68 (2014).

[36] O.D. Makinde, S. Khamis, M.S. Tshehla, and O. Franks, "Analysis of heat transfer in Berman flow of Nanofluids with Navier slip, viscous dissipation and convective cooling", Adv. Math. Phys. 2014 (4), 1-13 (2014). 